\title{
The interaction between a Three-Level Atom and Four Systems of N-Two Level Atoms
}

\author{
A. T. M. Makram-Allah", ${ }^{1,}$, M. M. A. Ahmed ${ }^{2}$, and D. A. M. Abo-Kahla ${ }^{3}$ \\ ${ }^{1}$ Department of Mathematics, Faculty of Women for Art, Science and Education, Ain Shams \\ University, Cairo, Egypt. \\ ${ }^{2}$ Department of Mathematics, Faculty of Science, Al-Azhar University, Nasr City, Cairo, Egypt. \\ ${ }^{3}$ Department of Mathematics, Faculty of Education, Ain Shams University, Roxy, Cairo, Egypt.
}

\begin{abstract}
We have four systems, each of them contains $\mathrm{N}$-atoms, and each atom of them consists of two levels, and all of them interact with an atom consisting of three levels. Moreover, the atom is also related to the systems by coupling parameters that are time-dependent. By considering particular conditions, the exact solution for the wave function is realized. Then we survey the atomic population inversion in addition to the normalized correlation functions. We examine the influence of many parameters on the previous statistical aspects. We deduce from the conclusions that the behavior of the correlation function is affected by the existence of the time-dependent coupling parameters between spins. We prove that changing the values of the coupling parameters between spins $\lambda \mathrm{k}$, the parameters $\varepsilon$, and the quantum numbers $\mathrm{N} \gamma$ enables us to control the correlated behavior in addition to the atomic population. So, we can control this model through these parameters.
\end{abstract}

keywords: Atom-Atom Interaction; Normalized Correlation Function; Non-Classical Behavior; Three-Level Atom.

\section{Introduction}

It is well known that the quantum mechanical atom-atom interaction can predict new aspects of the quantum nature of the system and the atom. There are a lot of models in quantum optics that describe the atom-atom interaction. For example, asingle two-level atom interacts with Ntwo-level atoms with a magnetic field effect that has been discussed in Ref. [1-2] and other cases, such as Ref. [3-9]. Recently, much attention has focused on the influence of the timedependent coupling parameters on various types of interactions. In the case of the atom-field interaction, the influence of time-dependent coupling parameters of the dimer system, has been discussed in Ref. [10]. Also, a three level $\Lambda$-type atom interacts with a two-mode with the existence of nonlinearity that has been discussed in Ref. [11-12] and [13-14]. On the other hand, in the atom-atom interaction, the effect of time-dependent coupling parameters of the model like our model, but for different cases from us that have been discussed recently in Ref. [15]. One of the most important goals of this paper is to survey the effect of atom-atom *Corresponding author: A. T. M. Makram-Allah, Department of Mathematics, Faculty of Women for Art, Science and Education, Ain Shams University, Cairo, Egypt.

E-mail: asmaa.tarek@,women.asu.edu.eg 
interaction on the atomic population inversion as well as the second-order correlation function. There are some examples of the atomic inversion evolution in the case of a two-level atom interacts with a various field, which has been discussed in Ref. [16 - 17]. Faraji et al. investigated the system of two-level atoms interacts with two fields by taking into account the dipole-dipole interaction of the atoms, and the time evolution of atomic inversion [18]. Faghihi et al., on the other hand, discussed the atomic inversion evolution of the system of a three-level atom of $\Lambda$-type interacts with a two-mode field in the existence of a bichromatic cavity [19]. Different types of interactions have been discussed in this field, for example, Ref. [20- 26]. Also, As the second-order function was evaluated, the system's non-classical behavior was better understood [27]- [32]. Dibakar et al. discussed many non-classical characteristics, like the sub-Poissonian statistics, correlation function among the two modes in Ref. [33]. AbdelWahab et al. examined the non-classical characteristic of a two two-level atoms that interacts with a two-mode cavity field [34]. There are different papers for several interactions and cases that have discussed the atomic inversion evolution and the second-order function as Ref. [3541].

In our previous paper [15], we studied the problem of four systems, each of them contains $\mathrm{N}$-atoms, and each atom of them consists of two levels, and all of them interact with an atom consisting of three levels. Moreover, the atom is also related to the systems by coupling parameters that are time-dependent. We also investigated the influences of the quantum numbers $N_{\gamma}(\gamma=1,2,3,4)$, the coupling parameters between spins $\lambda_{k}(k=1,2)$, the parameter $\varepsilon$, and some initial cases on the previous statistical aspects.

In this paper, we explore the previous model, but for different Hamiltonian, and cases.

We arrange the paper in the following organization: In Section 2, we describe the model of the system and obtain the exact solution for the model describing the interaction between a three-level atom interacting with four systems of $\mathrm{N}$-two level atoms with time-dependent coupling parameters, by taking some special cases. In section 3, and 4, we are investigating numerically the influences of the quantum numbers $N_{\gamma}(\gamma=1,2,3,4)$, the coupling parameters between spins $\lambda_{k}(k=1,2)$, the parameter $\varepsilon$, and the initial state on the previous statistical aspects. The conclusions are presented in section 5 .

\section{The Model}

The following Hamiltonian explains our model as ( we take $\hbar=1$ ): 


$$
\begin{aligned}
H=\sum_{\gamma=1}^{\gamma=4} \eta_{\gamma} J_{z}^{(\gamma)}+\sum_{l=1}^{l=3} \Omega_{l} S_{l l} & +\lambda_{1}(\tau)\left[S_{21} J_{+}^{(1)} J_{+}^{(2)}+J_{-}^{(2)} J_{-}^{(1)} S_{12}\right] \\
& +\lambda_{2}(\tau)\left[S_{32} J_{+}^{(3)} J_{+}^{(4)}+J_{-}^{(4)} J_{-}^{(3)} S_{23}\right]
\end{aligned}
$$

Where $\eta_{\gamma}, \gamma=1,2,3,4$ are the frequencies of the systems, $\Omega_{l}, l=1,2,3$ are the frequencies of the atom (where $\Omega_{1}>\Omega_{2}>\Omega_{3}$ ).

The atom coupling with the system by time-dependent coupling parameters $\lambda_{k}(\tau)(k=1,2)$ which given by:

$$
\lambda_{k}(\tau)=\lambda_{k} \cos \varepsilon \tau, k=1,2
$$

Also, $S_{\alpha \beta}=|\alpha\rangle\langle\beta|$ are the operators satisfy the commutation relation:

$$
\left[S_{\alpha \beta}, S_{i j}\right]=S_{\alpha j} \delta_{i \beta}-S_{i \beta} \delta_{\alpha j}
$$

The angular momentum $J_{ \pm}^{(\gamma)}$ and $J_{z}^{(\gamma)}$ satisfy the relations:

$$
\begin{gathered}
J_{L}^{(\gamma)}=\frac{1}{2} \sum_{K_{\gamma}=1}^{N_{\gamma}} \sigma_{L}^{K_{\gamma}}, L=x, y, z, \\
J_{ \pm}^{(\gamma)}=J_{x}^{(\gamma)} \pm i J_{y}^{(\gamma)} \\
{\left[J_{+}^{(\gamma)}, J_{-}^{(l)}\right]=2 J_{z}^{(\gamma)} \delta_{\gamma l}} \\
{\left[J_{z}^{(\gamma)}, J_{ \pm}^{(l)}\right]= \pm J_{ \pm}^{(\gamma)} \delta_{\gamma l}}
\end{gathered}
$$

And

$$
\begin{aligned}
& J_{ \pm}^{(\gamma)}\left|\zeta_{\gamma}, N_{\gamma}\right\rangle=\sqrt{\left(\zeta_{\gamma} \bar{\mp} N_{\gamma}\right)\left(\zeta_{\gamma} \pm N_{\gamma}+1\right)}\left|\zeta_{\gamma}, N_{\gamma} \pm 1\right\rangle \\
& J_{ \pm}^{(\gamma)}\left|\zeta_{\gamma}, N_{\gamma}\right\rangle=N_{\gamma}\left|\zeta_{\gamma}, N_{\gamma}\right\rangle
\end{aligned}
$$

We assume the state of our model to be in the form:

$$
\begin{aligned}
|W(\tau)\rangle= & G_{1}(\tau)\left|1, \zeta_{1}, N_{1}, \zeta_{2}, N_{2}, \zeta_{3}, N_{3}, \zeta_{4}, N_{4}\right\rangle \\
& +G_{2}(\tau)\left|2, \zeta_{1}, N_{1}+1, \zeta_{2}, N_{2}+1, \zeta_{3}, N_{3}, \zeta_{4}, N_{4}\right\rangle \\
+ & G_{3}(\tau)\left|3, \zeta_{1}, N_{1}+1, \zeta_{2}, N_{2}+1, \zeta_{3}, N_{3}+1, \zeta_{4}, N_{4}+1\right\rangle
\end{aligned}
$$

The Schrodinger equation is given by:

$$
i \frac{\partial}{\partial \tau}|W(\tau)\rangle=H|W(\tau)\rangle .
$$

From equations (1), (10), and (11), we have the following differential equations: 


$$
\begin{gathered}
i \frac{d G_{1}(\tau)}{d \tau}=\beta_{1} G_{1}(\tau)+\Gamma_{1} \cos \varepsilon \tau G_{2}(\tau), \\
i \frac{d G_{2}(\tau)}{d \tau}=\beta_{2} G_{2}(\tau)+\Gamma_{1} \cos \varepsilon \tau G_{1}(\tau)+\Gamma_{2} \cos \varepsilon \tau G_{3}(\tau) \\
i \frac{d G_{3}(\tau)}{d \tau}=\beta_{3} G_{3}(\tau)+\Gamma_{2} \cos \varepsilon \tau G_{2}(\tau)
\end{gathered}
$$

Where,

$$
\begin{gathered}
\beta_{1}=\eta_{1} N_{1}+\eta_{2} N_{2}+\eta_{3} N_{3}+\eta_{4} N_{4}+\Omega_{1} \\
\beta_{2}=\eta_{1}\left(N_{1}+1\right)+\eta_{2}\left(N_{2}+1\right)+\eta_{3} N_{3}+\eta_{4} N_{4}+\Omega_{2} \\
\beta_{3}=\eta_{1}\left(N_{1}+1\right)+\eta_{2}\left(N_{2}+1\right)+\eta_{3}\left(N_{3}+1\right)+\eta_{4}\left(N_{4}+1\right)+\Omega_{3} \\
\Gamma_{1}=\lambda_{1} \sqrt{\left(\zeta_{1}-N_{1}\right)\left(\zeta_{1}+N_{1}+1\right)\left(\zeta_{2}-N_{2}\right)\left(\zeta_{2}+N_{2}+1\right)} \\
\Gamma_{2}=\lambda_{2} \sqrt{\left(\zeta_{3}-N_{3}\right)\left(\zeta_{3}+N_{3}+1\right)\left(\zeta_{4}-N_{4}\right)\left(\zeta_{4}+N_{4}+1\right)}
\end{gathered}
$$

Now, we can solve the system (12), by these steps:

$$
\dot{G}(\tau)=F(\tau) G(\tau)
$$

Where

$$
G(\tau)=\left(\begin{array}{l}
G_{1} \\
G_{2} \\
G_{3}
\end{array}\right), F(\tau)=\left(\begin{array}{ccc}
-i \beta_{1} & -i \Gamma_{1} \cos \varepsilon \tau & 0 \\
-i \Gamma_{1} \cos \varepsilon \tau & -i \beta_{2} & -i \Gamma_{2} \cos \varepsilon \tau \\
0 & -i \Gamma_{2} \cos \varepsilon \tau & -i \beta_{3}
\end{array}\right)
$$

By considering particular conditions, the matrix $F(\tau)$ of the system is symmetric and the product of the matrix and the integral of this matrix is commutative. Therefore, the fundamental matrix of the system is given by:

$$
Q(\tau)=\exp \left(\int_{0}^{\tau} F(\tau) d \tau\right)
$$


We evaluate the matrix exponential by converting the matrix to diagonal form, also we compute the eigenvalues and eigenvectors of the matrix $\left(\int_{0}^{\tau} F(\tau) d \tau\right)$. Then, we get the transition matrix $(M)$ of the eigenvectors. The Jordan form $R$ of the matrix can be written as:

$$
R=M^{-1}\left[\int_{0}^{\tau} F(\tau) d \tau\right] \mathrm{M}
$$

Therefore, we can compute the fundamental matrix as:

$$
Q(\tau)=M^{-1} \exp (R) M
$$

Then the solution become $G(\tau)=Q(\tau) C$, where $C=G(0)$. From the initial conditions, the unknown vector $G(\tau)$ can be obtained, then the nonhomogeneous system (12) can be solved. Then, we get the solution of the system as follows:

$$
\begin{gathered}
G_{1}(\tau)=\frac{e^{-i \beta_{1} \tau}}{v^{2}}\left\{\left(\Gamma_{1}^{2} \cos \theta+\Gamma_{2}^{2}\right) G_{1}(0)-i v \Gamma_{1} \sin \theta G_{2}(0)\right. \\
\left.+\Gamma_{1} \Gamma_{2}(\cos \theta-1) G_{3}(0)\right\}, \\
G_{2}(\tau)=-\frac{e^{-i \beta_{1} \tau}}{v}\left\{i \sin \theta\left(\Gamma_{1} G_{1}(0)+\Gamma_{2} G_{3}(0)\right)-v \cos \theta G_{2}(0)\right\}, \\
G_{3}(\tau)=\frac{e^{-i \beta_{1} \tau}}{v^{2}}\left\{(\cos \theta-1) \Gamma_{1} \Gamma_{2} G_{1}(0)-i v \Gamma_{2} \sin \theta G_{2}(0)\right. \\
\left.\quad++\left(\Gamma_{2}^{2} \cos \theta+\Gamma_{1}^{2}\right) G_{3}(0)\right\},
\end{gathered}
$$

Where

$$
\begin{aligned}
& \theta=v \epsilon(\tau), \\
& \epsilon(\tau)=\frac{\sin (\varepsilon \tau)}{\varepsilon}, \\
& v=\sqrt{\Gamma_{1}^{2}+\Gamma_{2}^{2}} .
\end{aligned}
$$




\section{The Atomic Inversion}

The atomic inversion can be written as follows [42]- [45]:

$$
\left\langle S_{z}\right\rangle=\left|G_{1}(\tau)\right|^{2}+\left|G_{2}(\tau)\right|^{2}-\left|G_{3}(\tau)\right|^{2},
$$

To discuss the atomic inversion, we have plotted several figures in which we take into account $\zeta_{1}=50, \zeta_{2}=40, \zeta_{3}=30, \zeta_{4}=20$, and various values of $\lambda_{k}, N_{\gamma}, \varepsilon$, also at initially the state be $\{\mathrm{i}\}|W(0)\rangle_{r}=|1\rangle,\{$ ii $\}|W(0)\rangle_{r}=\frac{1}{\sqrt{2}}|1\rangle+\frac{1}{\sqrt{2}}|2\rangle$, and $\{$ iii $\}|W(0)\rangle_{r}=\frac{1}{\sqrt{2}}|1\rangle+$ $\frac{1}{2}|2\rangle+\frac{1}{2}|3\rangle$. We show that in all figures the oscillations of the atomic inversion curve are periodic. In Fig. 1, we modify the values of the coupling parameters $\lambda_{k}$ and make the other parameters fixed i.e. $N_{\gamma}=1, \varepsilon=1$, also at initially state be $\{\mathrm{i}\}$ to examine their influences on the atomic inversion. We note that the number of oscillations rises and the maximum value, $\left\langle S_{z}\right\rangle=$ 1 , reductions to attain its minimum value, $\left\langle S_{z}\right\rangle=-0.2$, as the coupling parameters $\lambda_{k}$ rise. We have at the top of the curve constant interval at $\lambda=0.25$ decay as the parameters $\lambda_{k}$ rise. In Figs. 2(a-c), we modify the values of the quantum numbers $N_{\gamma}$ and make the other parameters fixed i.e. $\lambda_{k}=1, \varepsilon=1$, to examine their influences on the atomic inversion. As the quantum numbers, $N_{\gamma}$ raise the number and the phase of oscillations reduction. In Fig. 2(a), initially state be $\{\mathrm{i}\}$, but in Fig. 2(b), initially state be $\{$ ii $\}$. In both, the curve begins at the highest point, $\left\langle S_{z}\right\rangle=1$, this high point reductions to attain the lowest point, $\left\langle S_{z}\right\rangle=0.3$, in Fig. 2(a) at $N_{\gamma}=1$. On the other hand, in Fig. 2(b), the high point reductions to attain the lowest point but at $\left\langle S_{z}\right\rangle=0.7$, at $N_{\gamma}=1$. As the quantum numbers $N_{\gamma}$ rise, the lowest point rises to attain $\left\langle S_{z}\right\rangle \simeq 0.9$ at $N_{\gamma}=19$, in Fig. 2(a). Also in Fig. 2(b), as the quantum numbers $N_{\gamma}$ rise, the lowest point rises to be closed too 1. But in Fig. 2(c), initially state be $\{$ iii $\}$, the curve begins at the lowest point, $\left\langle S_{z}\right\rangle=0.5$, this lowe point rises to attain the highest point, $\left\langle S_{z}\right\rangle=1$, at $N_{\gamma}=1$. As the quantum numbers $N_{\gamma}$ rise, the maximum value reduction to attain $\left\langle S_{z}\right\rangle=0.7$ at $N_{\gamma}=19$. In Figs. 3(a-c), we modify the value of the parameter $\varepsilon$ and make the other parameters fixed i.e. $\lambda_{k}=1, N_{\gamma}=1$, to examine their influences on the atomic inversion. As the parameter $\varepsilon$ rises the values rise, but phase of oscillations reductions. In Fig. 3(a), initially state be $\{i\}$, the curve begins at the highest point, $\left\langle S_{z}\right\rangle=1$, this high point reductions to attain the lowest point, $\left\langle S_{z}\right\rangle \simeq 0.35$. As the parameter $\varepsilon$ rises, the lowest point rises to attain $\left\langle S_{z}\right\rangle \simeq 0.65$. Also in Fig. 3(b), initially state be $\{$ ii $\}$, the curve begins at the highest point, $\left\langle S_{z}\right\rangle=1$, this the high point reductions to attain the lowest point, $\left\langle S_{z}\right\rangle \simeq 0.65$. As the parameter $\varepsilon$ rises, the lowest point rises to attain $\left\langle S_{z}\right\rangle \simeq 0.75$. But in 
Fig. 3(c), initially state be $\{$ iii $\}$ the curve begins at the lowest point, $\left\langle S_{z}\right\rangle=0.5$, this lowest point rises to attain the highest point, $\left\langle S_{z}\right\rangle=1$, As the parameter $\varepsilon$ rises, the highest point reduction to attain $\left\langle S_{z}\right\rangle \simeq 0.95$.

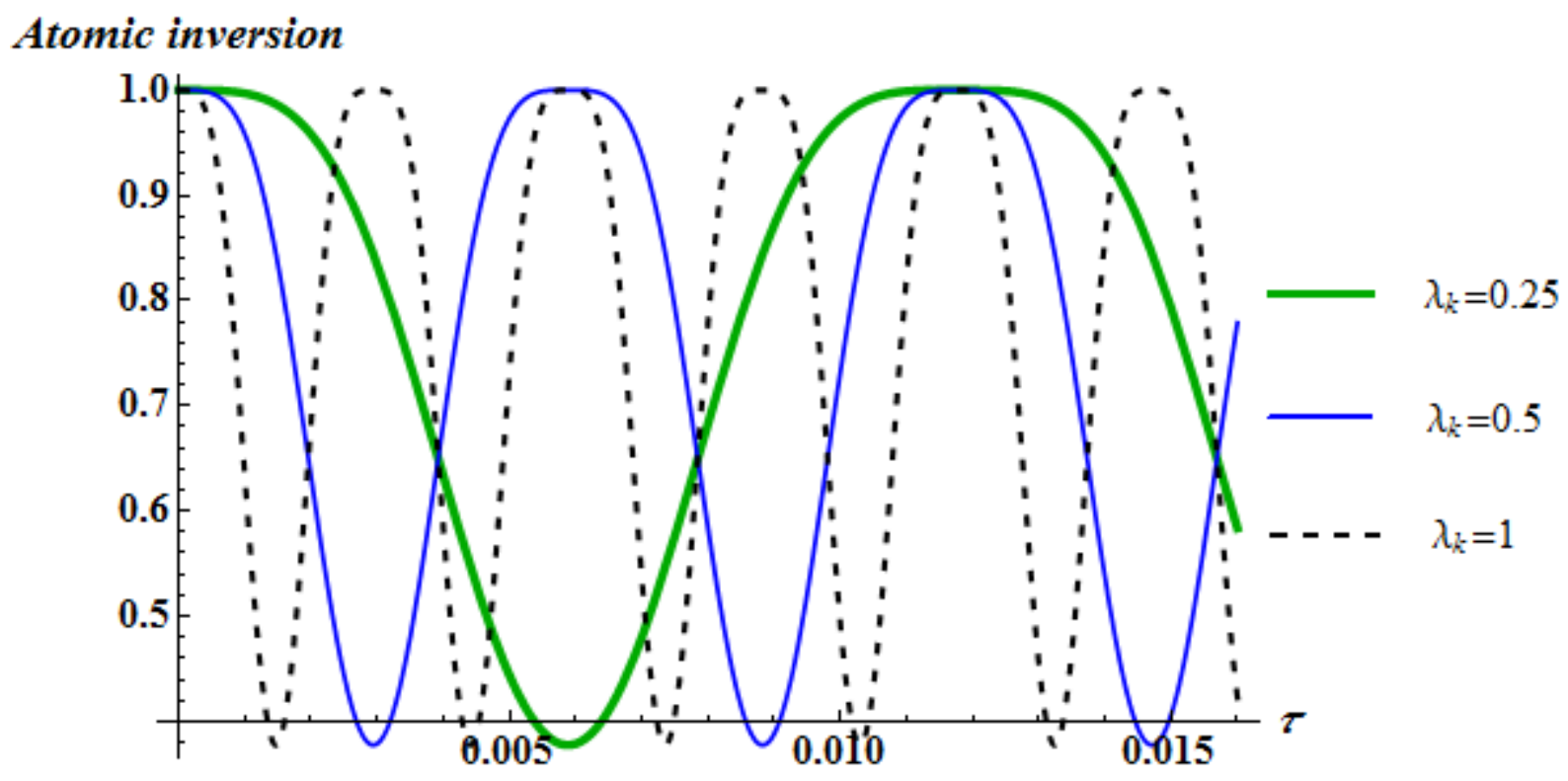

Fig. 1: The atomic inversion evolution at initially the state be $\{i\}$ with $\mathrm{N}_{\gamma}=1$, and $\varepsilon=1$.

Atomic inversion

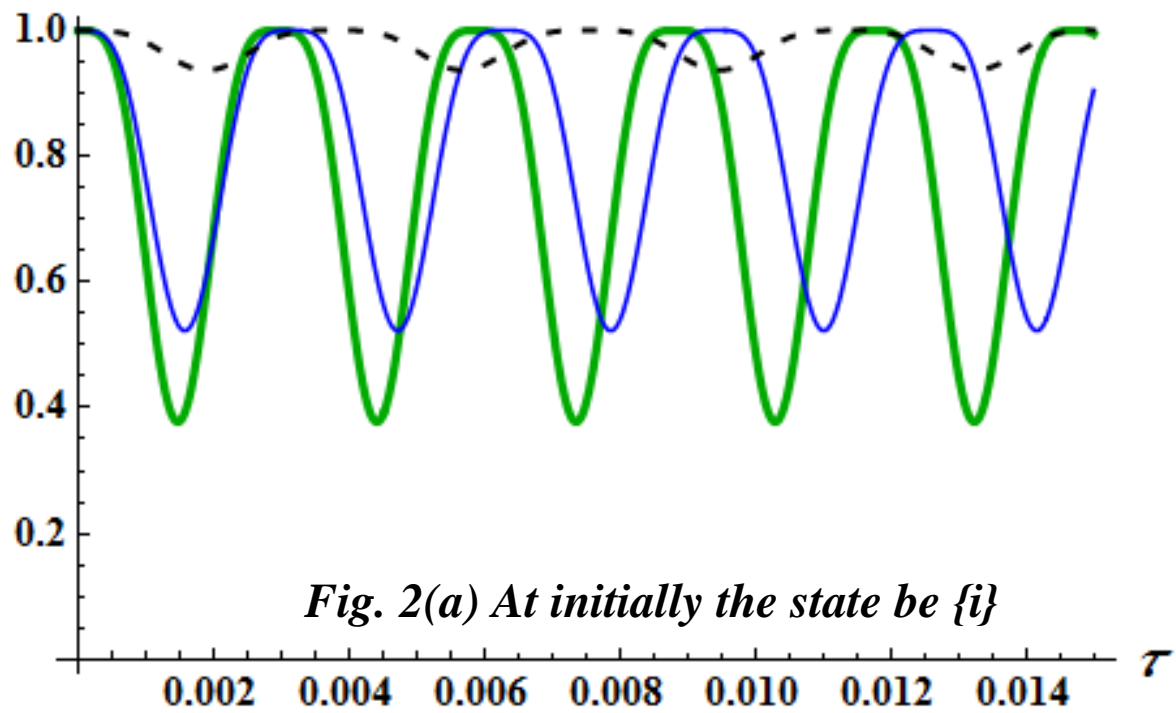


Atomic inversion

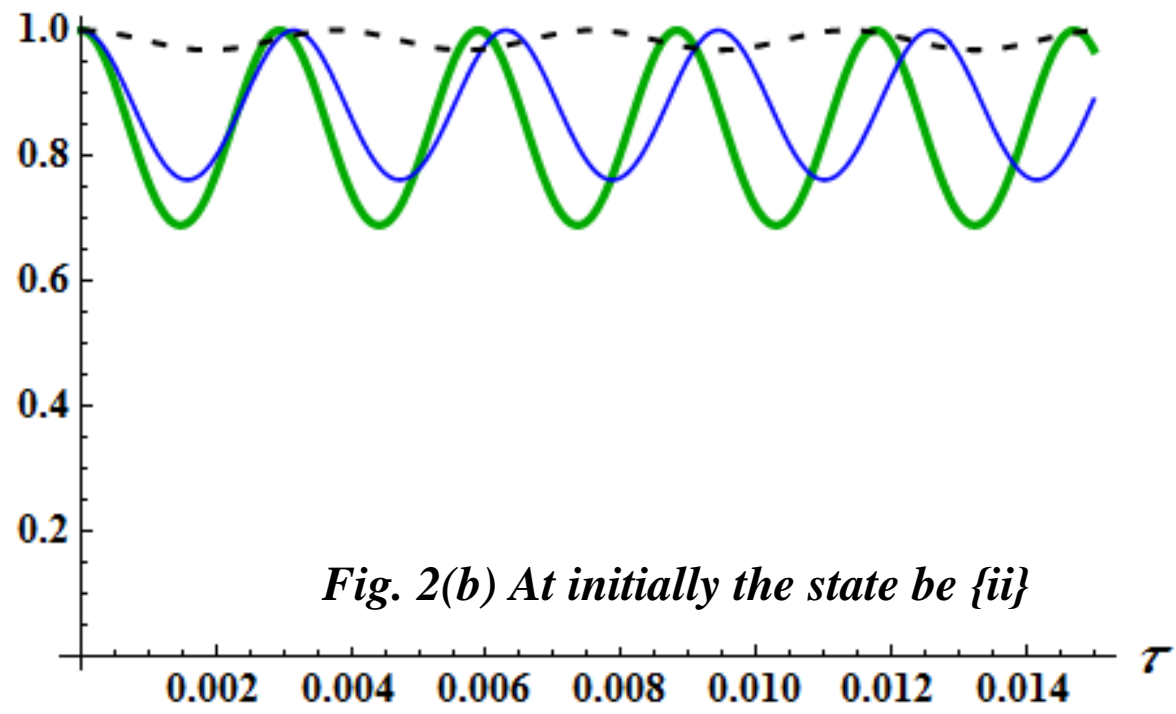

Atomic inversion

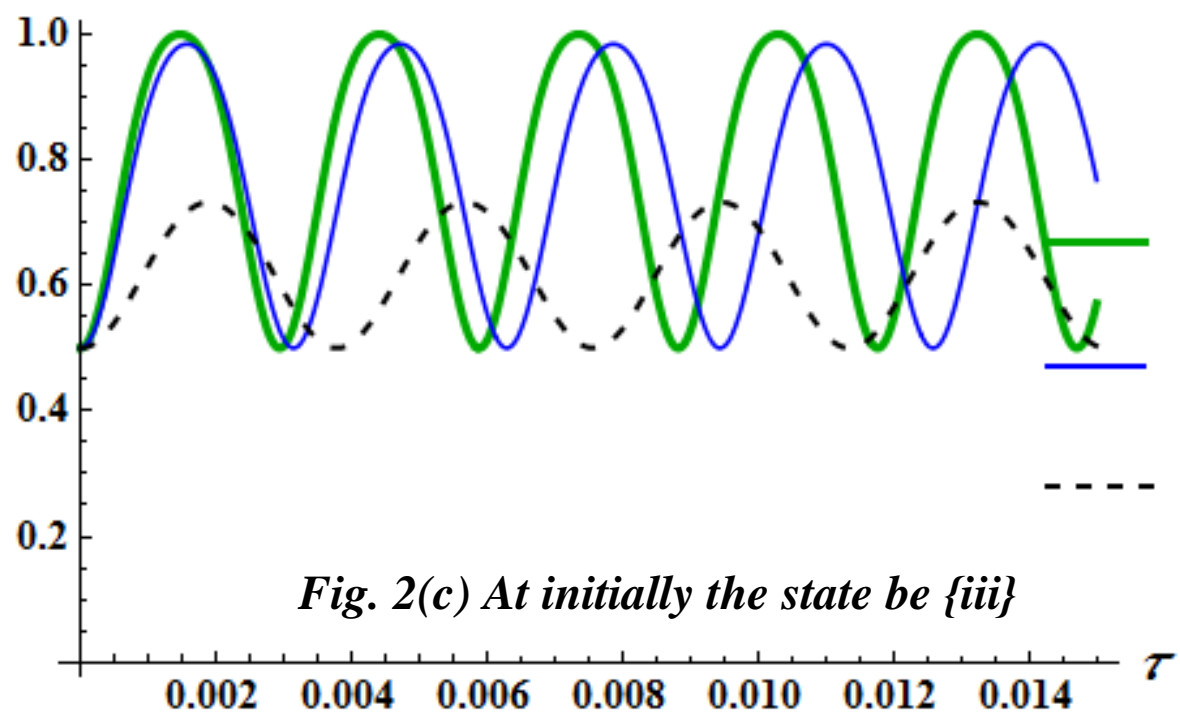

Figs. 2: The figures for $\lambda_{k}=1$, and $\varepsilon=1$. 
Atomic inversion

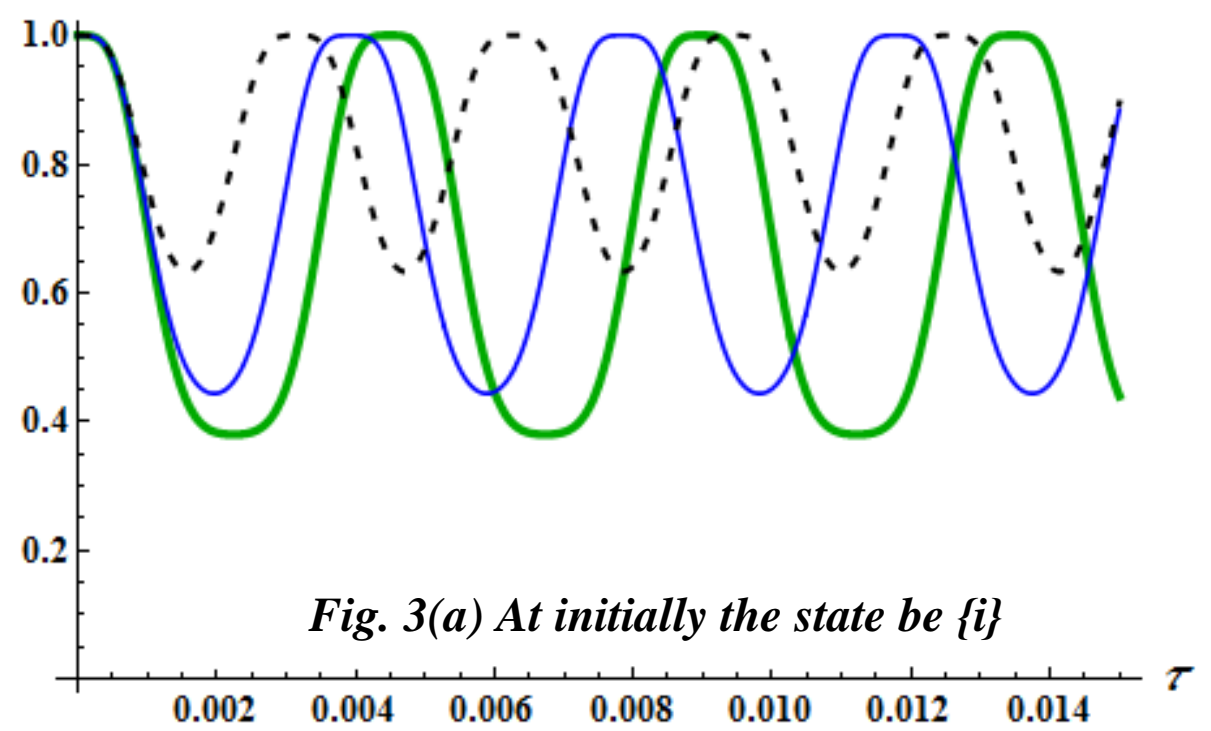

Atomic inversion

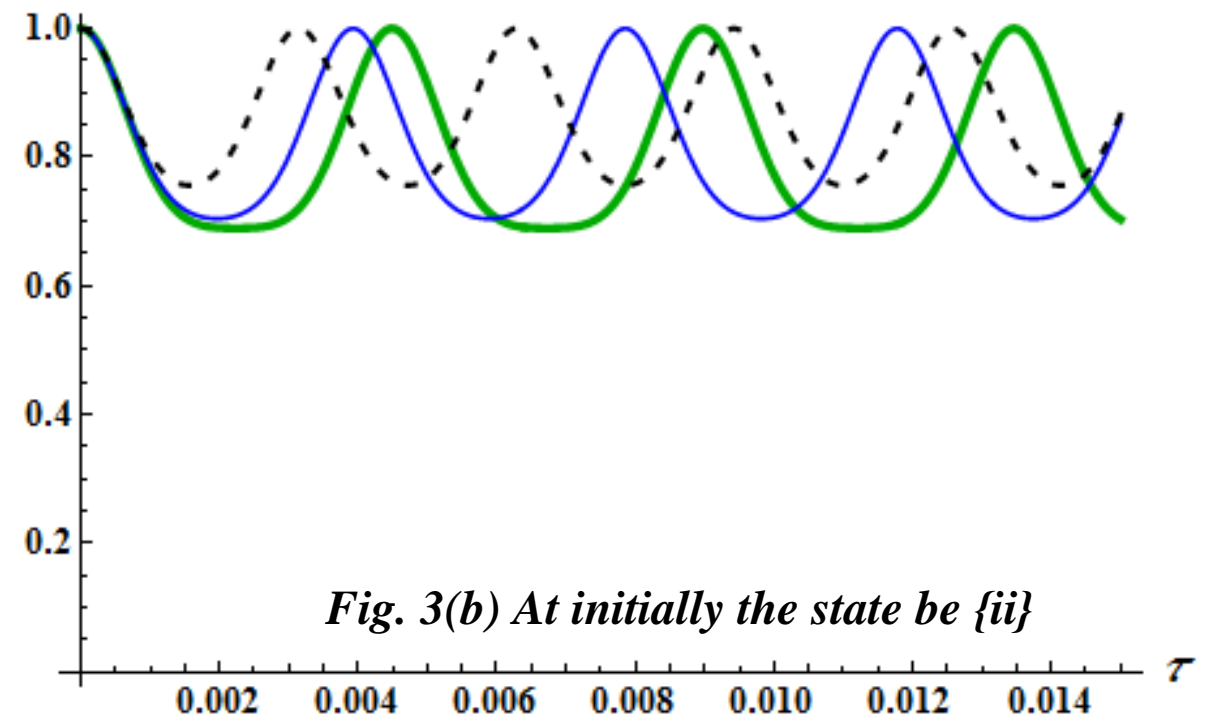




\section{Atomic inversion}

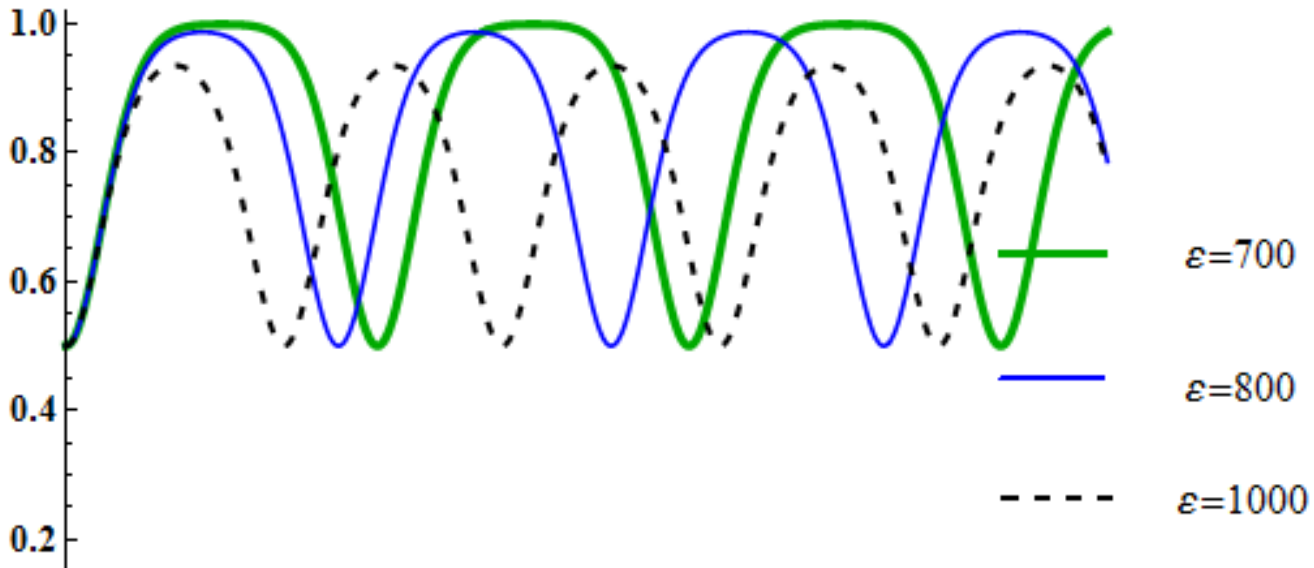

Fig. 3(c) At initially the state be \{iii\}

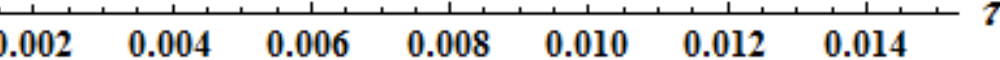

Figs. 3: The figures for $\lambda_{k}=1$, and $N_{\gamma}=1$.

\section{The Correlation Function}

The normalized second-order correlation function can be written as [2], [15], [24] and [46]:

$$
g_{\beta}^{2}(\tau)=\frac{\left\langle J_{+}^{(\beta) 2} J_{-}^{(\beta) 2}\right\rangle}{\left\langle J_{+}^{(\beta)} J_{-}^{(\beta)}\right\rangle^{2}}, \quad \beta=1,2,3,4
$$

Then we evaluate these expectation values, where

$$
\begin{aligned}
& J_{+}^{(\beta) 2} J_{-}^{(\beta) 2}=\varphi\left(J_{z}^{(\beta)}-1\right) \varphi\left(J_{z}^{(\beta)}\right) \\
& J_{+}^{(\beta)} J_{-}^{(\beta)}=\varphi\left(J_{z}^{(\beta)}\right)
\end{aligned}
$$

Equation (31) becomes

$$
\begin{gathered}
g_{\beta}^{2}(\tau)=\frac{\left\langle\varphi\left(J_{z}^{(\beta)}-1\right) \varphi\left(J_{z}^{(\beta)}\right)\right\rangle}{\left\langle\varphi\left(J_{z}^{(\beta)}\right)\right\rangle^{2}} \\
\varphi\left(J_{z}^{(\beta)}\right)=J^{(\beta) 2}-J_{z}^{(\beta) 2}+J_{z}^{(\beta) 2} .
\end{gathered}
$$

By substituting from (10) in (31) and (32), we have:

$$
g_{1}^{2}(\tau)=\frac{\varphi\left(N_{1}, \zeta_{1}\right)\left\{\varphi\left(N_{1}-1, \zeta_{1}\right)\left|G_{1}(\tau)\right|^{2}+\varphi\left(N_{1}+1, \zeta_{1}\right)\left[\left|G_{2}(\tau)\right|^{2}+\left|G_{3}(\tau)\right|^{2}\right]\right\}}{\left\{\varphi\left(N_{1}, \zeta_{1}\right)\left|G_{1}(\tau)\right|^{2}+\varphi\left(N_{1}+1, \zeta_{1}\right)\left[\left|G_{2}(\tau)\right|^{2}+\left|G_{3}(\tau)\right|^{2}\right]\right\}^{2}},
$$




$$
\begin{aligned}
& g_{2}^{2}(\tau) \\
& =\frac{\varphi\left(N_{2}, \zeta_{2}\right)\left\{\varphi\left(N_{2}-1, \zeta_{2}\right)\left|G_{1}(\tau)\right|^{2}+\varphi\left(N_{2}+1, \zeta_{2}\right)\left[\left|G_{2}(\tau)\right|^{2}+\left|G_{3}(\tau)\right|^{2}\right]\right\}}{\left\{\varphi\left(N_{2}, \zeta_{2}\right)\left|G_{1}(\tau)\right|^{2}+\varphi\left(N_{2}+1, \zeta_{2}\right)\left[\left|G_{2}(\tau)\right|^{2}+\left|G_{3}(\tau)\right|^{2}\right]\right\}^{2}} \\
& g_{3}^{2}(\tau) \\
& =\frac{\varphi\left(N_{3}, \zeta_{3}\right)\left\{\varphi\left(N_{3}-1, \zeta_{3}\right)\left[\left|G_{1}(\tau)\right|^{2}+\left|G_{2}(\tau)\right|^{2}\right]+\varphi\left(N_{3}+1, \zeta_{3}\right)\left|G_{3}(\tau)\right|^{2}\right\}}{\left\{\varphi\left(N_{3}, \zeta_{3}\right)\left[\left|G_{1}(\tau)\right|^{2}+\left|G_{2}(\tau)\right|^{2}\right]+\varphi\left(N_{3}+1, \zeta_{3}\right)\left|G_{3}(\tau)\right|^{2}\right\}^{2}} \\
& g_{4}^{2}(\tau) \\
& =\frac{\varphi\left(N_{4}, \zeta_{4}\right)\left\{\varphi\left(N_{4}-1, \zeta_{4}\right)\left[\left|G_{1}(\tau)\right|^{2}+\left|G_{2}(\tau)\right|^{2}\right]+\varphi\left(N_{4}+1, \zeta_{4}\right)\left|G_{3}(\tau)\right|^{2}\right\}}{\left\{\varphi\left(N_{4}, \zeta_{4}\right)\left[\left|G_{1}(\tau)\right|^{2}+\left|G_{2}(\tau)\right|^{2}\right]+\varphi\left(N_{4}+1, \zeta_{4}\right)\left|G_{3}(\tau)\right|^{2}\right\}^{2}}
\end{aligned}
$$

Where

$$
\varphi\left(N_{\beta}, \zeta_{\beta}\right)=\left(\zeta_{\beta}+N_{\beta}\right)\left(\zeta_{\beta}-N_{\beta}+1\right), \beta=1,2,3,4
$$

We note that from pervious equations that $g_{1}^{2}(\tau)=g_{2}^{2}(\tau)$ also $g_{3}^{2}(\tau)=g_{4}^{2}(\tau)$. So, Figs. 4,5 and 6 show the normalized second-order correlation functions $g_{1}^{2}(\tau)$, and $g_{3}^{2}(\tau)$ only vs. the time $\tau$. To discuss the normalized second-order correlation functions we will plot several figures in which we take into account $\zeta_{\gamma}=\frac{5}{2}$, also at initially state be $\{\mathrm{i}\}$ and modify different values of $\lambda_{k}, N_{\gamma}$, and $\varepsilon$ parameters. We show that in all figures the oscillations of the secondorder correlation functions is periodic and regular. In Fig. 4, we modify the values of the coupling parameters $\lambda_{k}$ and make the other parameters fixed i.e. $N_{\gamma}=1$ and $\varepsilon=1$, to examine their influences on the correlation functions $g_{1}^{2}(\tau)$ and $g_{3}^{2}(\tau)$. The correlation functions $g_{1}^{2}(\tau), g_{3}^{2}(\tau)>1$, thus these functions show super-Poissonian behavior. In Fig. 5, we also modify the values of the quantum numbers $N_{\gamma}$ and make the other parameters fixed i.e. $\lambda_{k}=1$ and $\varepsilon=1$, to examine their influences on the correlation functions $g_{1}^{2}(\tau)$ and $g_{3}^{2}(\tau)$. The correlation functions $g_{1}^{2}(\tau)$ and $g_{3}^{2}(\tau)$ grow up As $N_{\gamma}$ rises. At $N_{\gamma}=\frac{-3}{2}, \frac{-1}{2}$ the functions $g_{1}^{2}(\tau)$ and $g_{3}^{2}(\tau)<1$, so these functions show sub-Poissonian behavior. But As $N_{\gamma}=\frac{1}{2}$, the correlation functions $g_{1}^{2}(\tau)$ and $g_{3}^{2}(\tau)$ sometimes show sub-Poissonian behavior ( $\left.g_{1}^{2}(\tau), g_{3}^{2}(\tau)<1\right)$ and sometimes show super-Poissonian behavior $\left(g_{1}^{2}(\tau), g_{3}^{2}(\tau)>1\right)$. As $N_{\gamma}=\frac{3}{2}$, the correlation functions $g_{1}^{2}(\tau)$ and $g_{3}^{2}(\tau)$ show super-Poissonian behavior $\left(g_{1}^{2}(\tau), g_{3}^{2}(\tau)>1\right)$. In Fig. 6, we modify the values of the parameter $\varepsilon$ and make the other parameters fixed i.e. $N_{\gamma}=1$ and $\lambda_{k}=1$, to examine their influences on the correlation functions $g_{1}^{2}(\tau)$ and $g_{3}^{2}(\tau)$. The correlation functions $g_{1}^{2}(\tau), g_{3}^{2}(\tau)>1$ at all values of the parameter $\varepsilon$, 
thus these functions show super-Poissonian behavior. We note that the number of oscillations rises as the parameter $\varepsilon$ increases, yet the phase of the oscillations reductions.

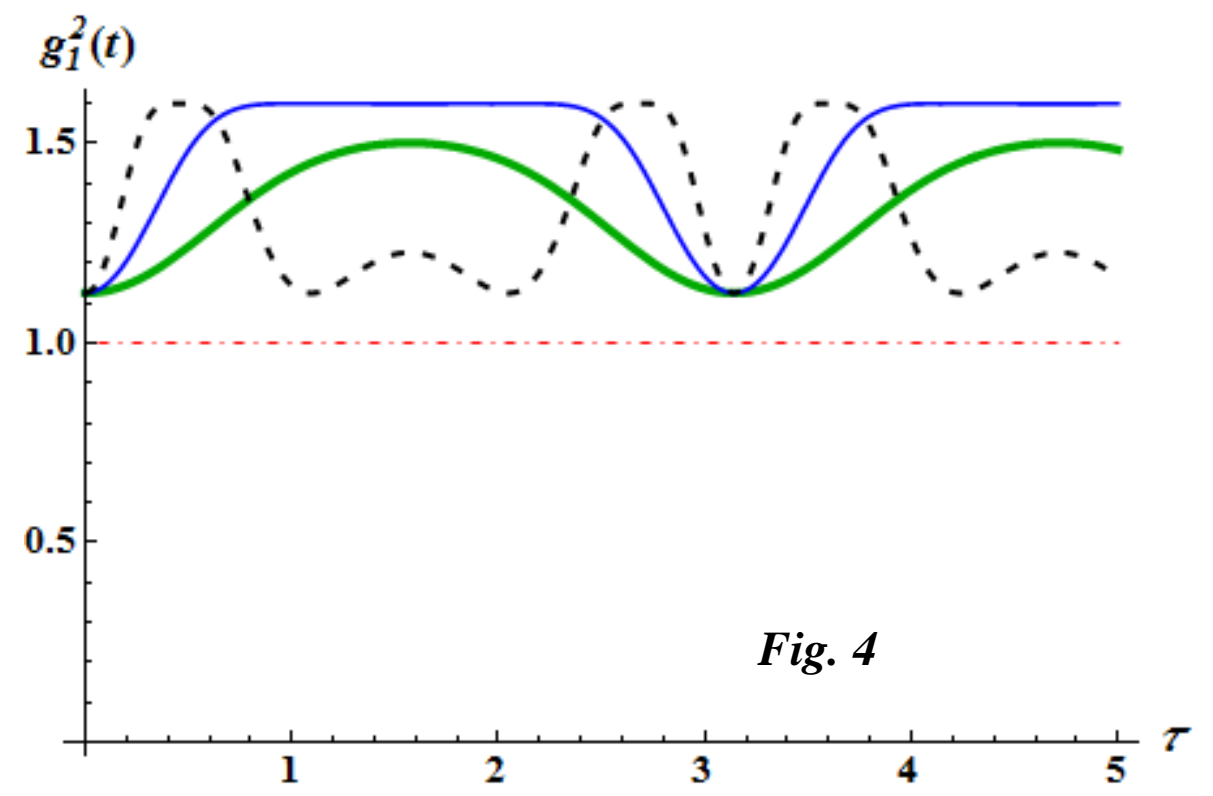




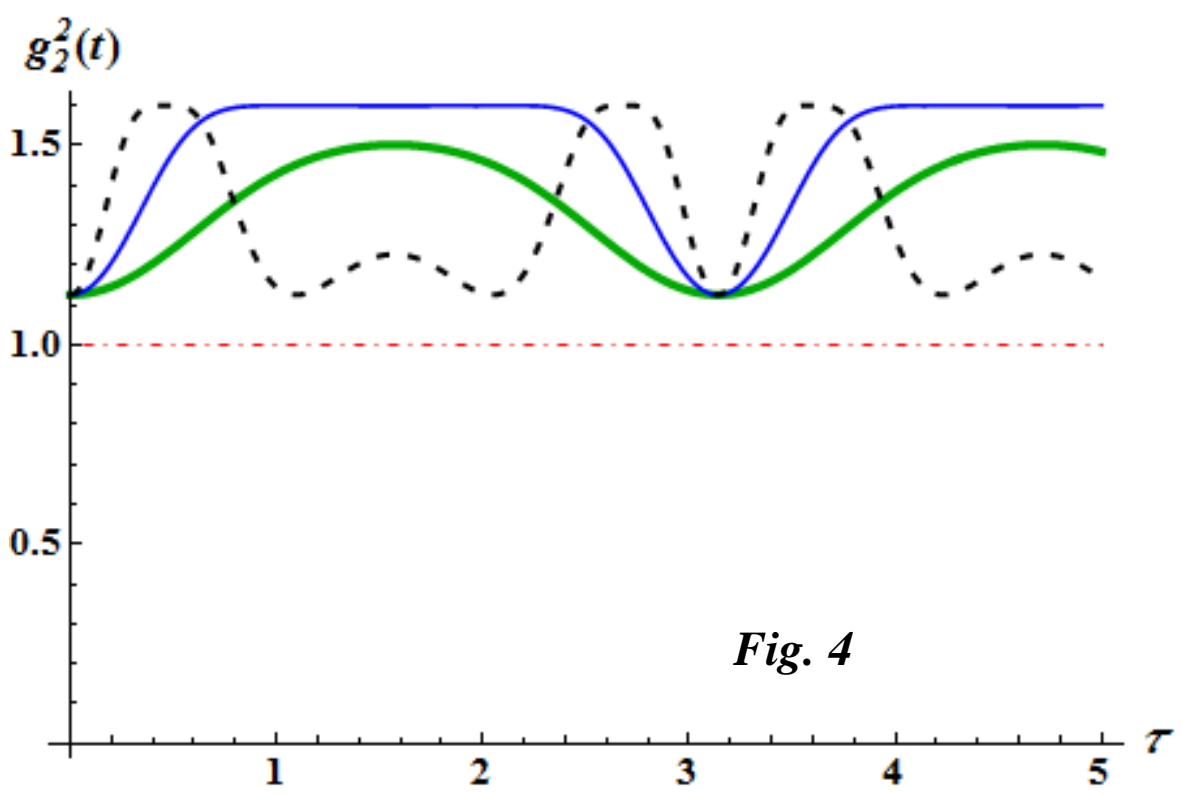

$g_{3}^{2}(t)$

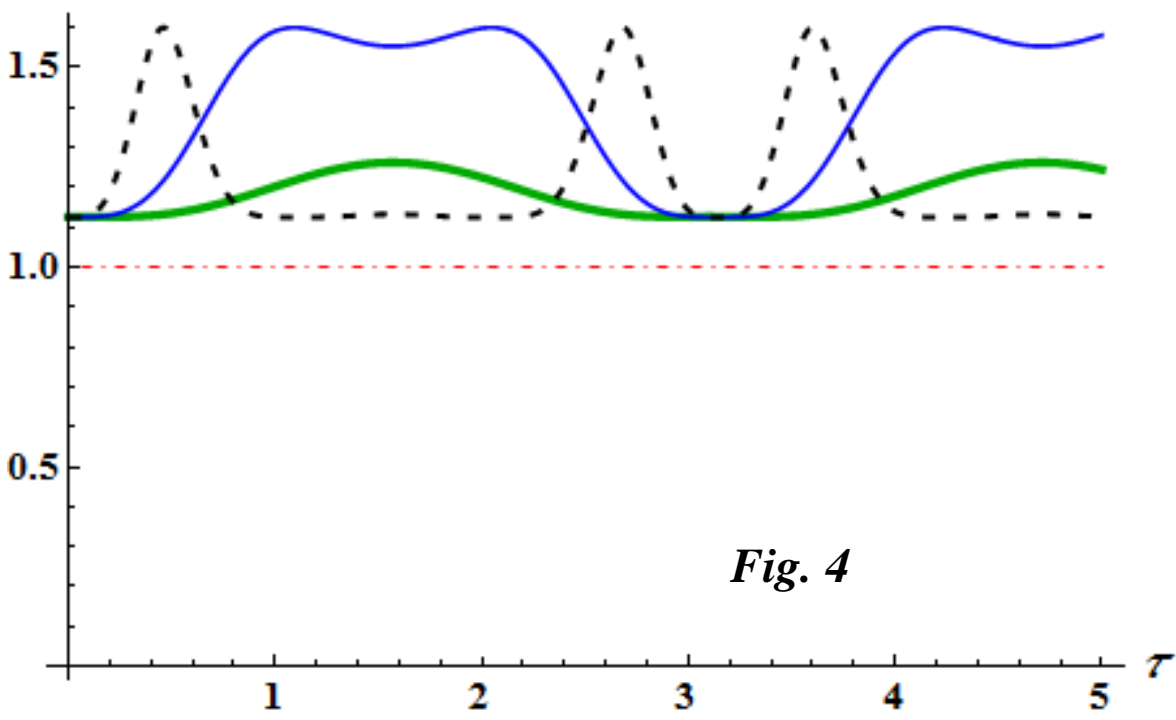




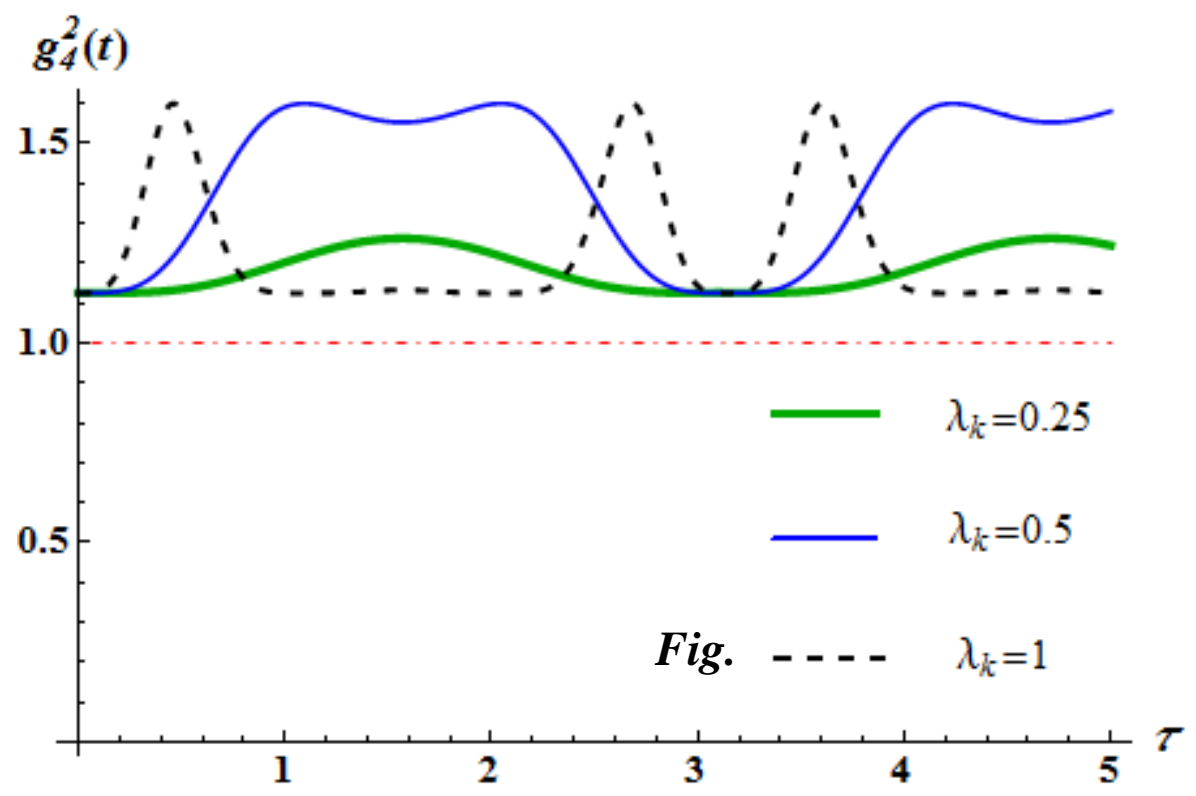

Figs. 4: The functions $g_{\beta}^{2}(\tau)$ vs. the time $\tau$. At initially the state be $\{\mathrm{i}\}$ with $N_{\gamma}=\frac{3}{2}, \varepsilon=1$

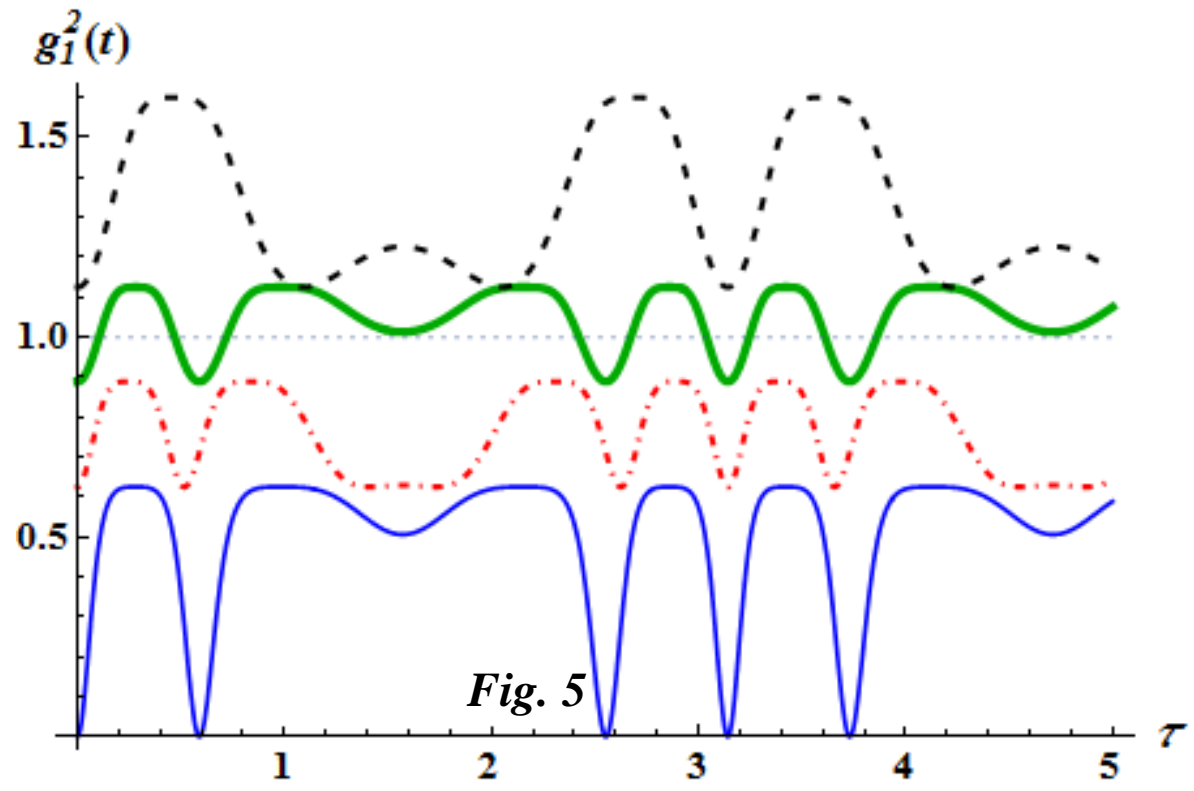




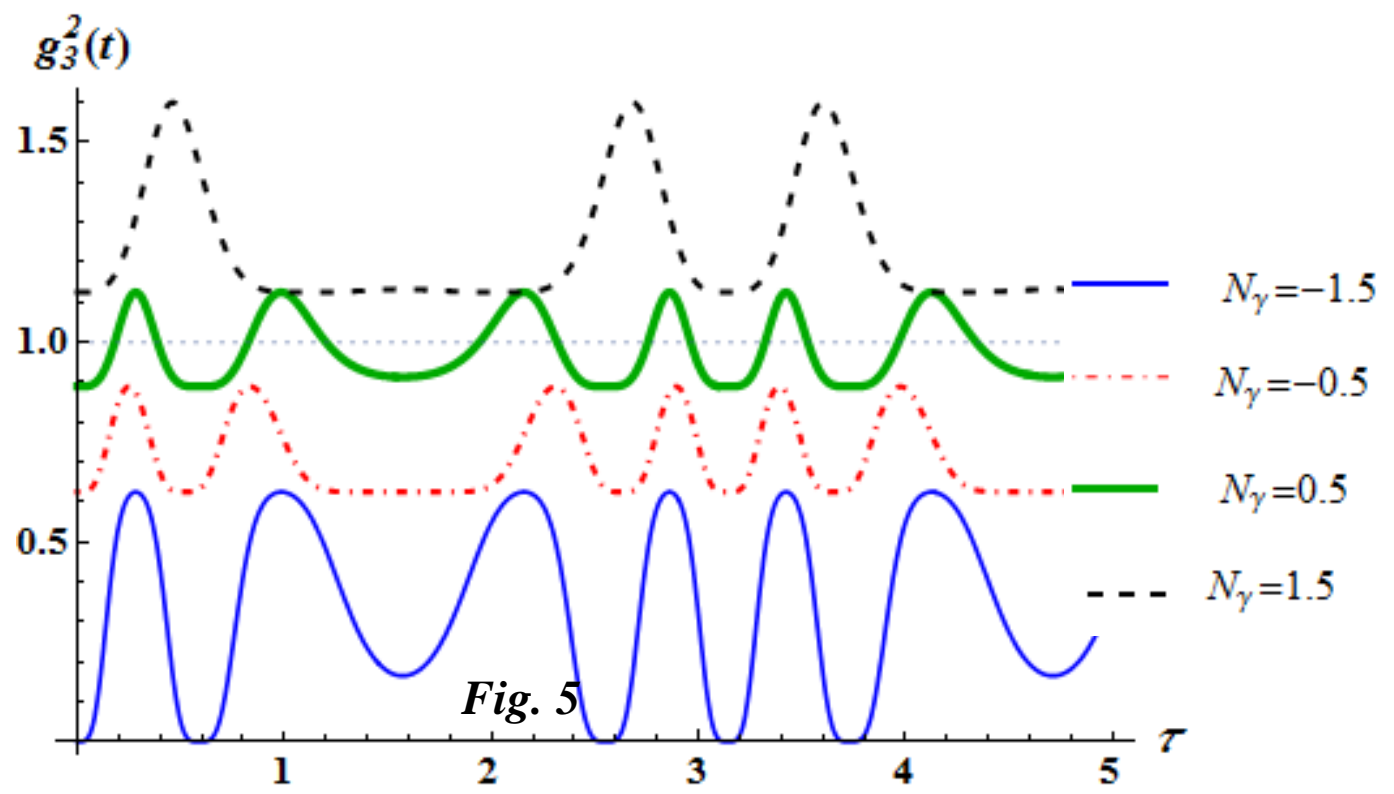

Figs. 5: The functions $g_{\beta}^{2}(\tau)$ vs. the time $\tau$. At initially the state be $\{\mathrm{i}\}$ with $\lambda_{K}=1, \varepsilon=1$.

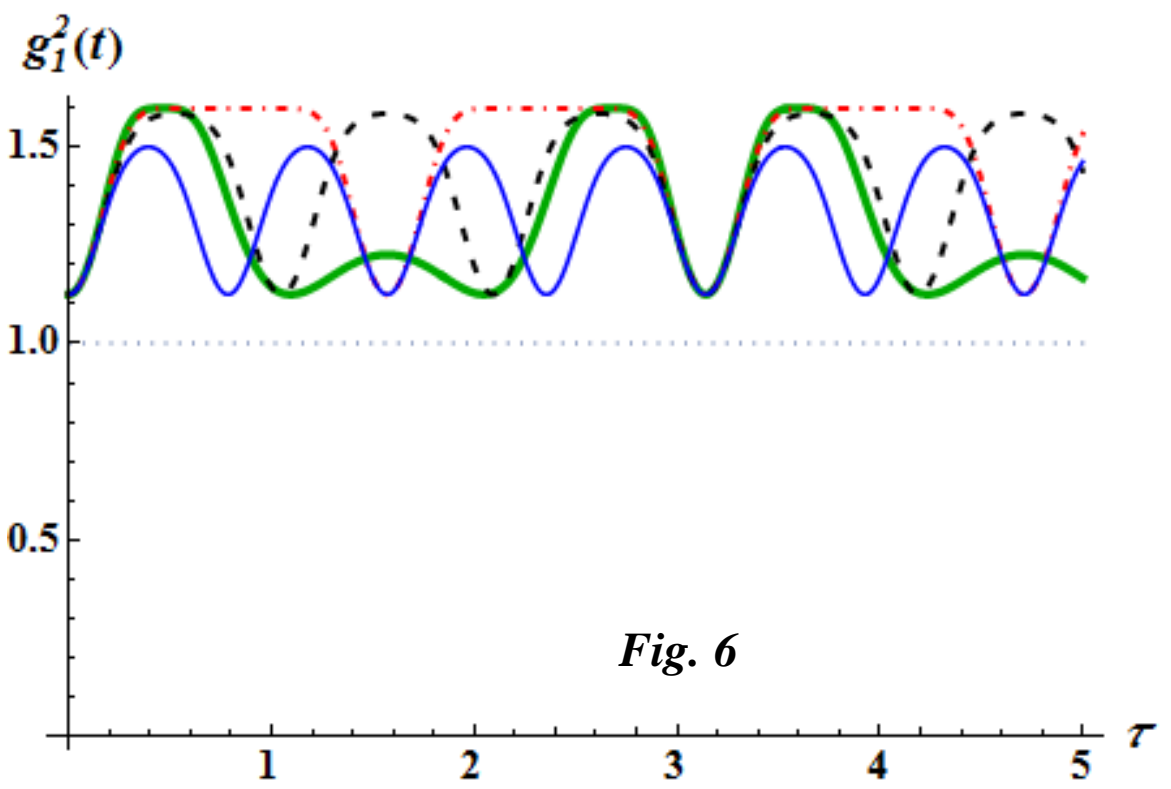




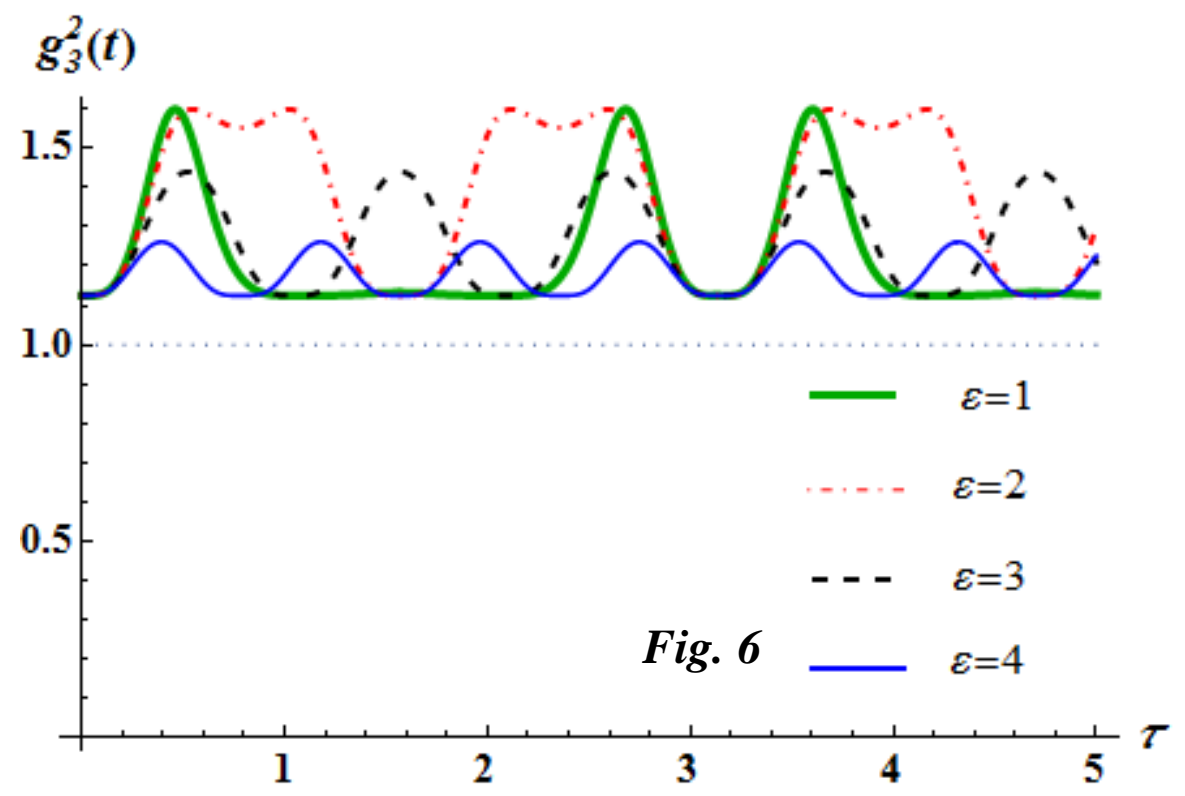

Figs. 6: The functions $g_{\beta}^{2}(\tau)$ vs. the time $\tau$. At initially the state be $\{\mathrm{i}\}$ with $N_{\gamma}=\frac{3}{2}, \lambda_{K}=1$

\section{Conclusion}

In this paper, we have studied four systems, each of them contains $\mathrm{N}$-atoms, and each atom of them consists of two levels, and all of them interact with an atom consisting of three levels. The coupling parameters between the atom and the systems are modulated to be timedependent. By considering particular conditions, the exact solution for this model has been obtained. Therefore, the wave function has been obtained as well. We also have discussed the influences of various parameters like for example the coupling parameters $\lambda_{k}$, the quantum numbers $N_{\gamma}$, and the (decay rates) parameter $\varepsilon$ on some statistical aspects. We have managed to study the atomic population inversion in addition to the second-order correlation function. In our handling of the atomic population inversion, we have realized that there is a constant interval in the top of the curve at $\lambda=0.25$, and then it decays and the maximum value, $\left\langle S_{z}\right\rangle=$ 1 , reductions to attain its minimum value, $\left\langle S_{z}\right\rangle=-0.2$, as the coupling parameters $\lambda_{k}$ rise. On the other hand, in Ref. [2-15], this constant interval also decays as the coupling parameters $\lambda$ rise and the number of periodic oscillations rises, also the maximum value, $\left\langle S_{z}\right\rangle=1$. When the coupling parameters $\lambda$ rise, the maximum value reductions to attain a different minimum value than our model, $\left\langle S_{z}\right\rangle=-0.75$, in Ref.[2], and, $\left\langle S_{z}\right\rangle=-0.4$, in Ref.[15]. This difference in the minimum value between the three cases can be assigned to a different number of systems and the Hamiltonian. We show that as the parameter $\varepsilon$ rises for all initial be $\{\mathrm{i}\},\{\mathrm{ii}\}$, and $\{\mathrm{iii}\}$, the 
values rise, but phase of oscillations reductions. For the first two initial states, the minimum value of the atomic population inversion curve rises to attain $\left\langle S_{z}\right\rangle \simeq 0.65$. But at the last initial state, the maximum value of the atomic population inversion curve reductions to attain $\left\langle S_{z}\right\rangle \simeq$ 0.95. Also, we note that the correlation function $g_{1}^{2}(\tau)=g_{2}^{2}(\tau)$ and also $g_{3}^{2}(\tau)=g_{4}^{2}(\tau)$. The correlation functions at all values of the parameter $\varepsilon$ and the coupling parameters $\lambda_{k}$, thus these functions show super-Poissonian behavior. We note that the number of oscillations rises as the parameter $\varepsilon$ rises, but the phase of the oscillations reductions. On the other hand, As changing the quantum numbers $N_{\gamma}$, the correlation functions $g_{1}^{2}(\tau)$ and $g_{3}^{2}(\tau)$ sometimes show subPoissonian behavior $\left(g_{1}^{2}(\tau), g_{3}^{2}(\tau)<1\right)$ and sometimes show super-Poissonian behavior ( $\left.g_{1}^{2}(\tau), g_{3}^{2}(\tau)>1\right)$. The correlation functions $g_{1}^{2}(\tau)$ and $g_{3}^{2}(\tau)$ grow up As $N_{\gamma}$ rises. Finally, we have proved that changing the values of the coupling parameters between spins $\lambda_{k}$, the parameters $\varepsilon$, and the quantum numbers $N_{\gamma}$ enables us to control the correlated behavior.

\section{Acknowledgments}

The authors appreciate deeply all the constructive opinions and sincere support of Professor Abdel-Shafy Fahmy Obada (A-S. F. Obada), mathematics department, Al-Azhar university, faculty of science.

\section{References}

[1] M. Sebawe Abdalla, M. M. A. Ahmed, R. N. Rabea, "Nonclassical Properties for Two Coupled N-Two-Level Atom and a Single Two-Level Atom Under an External Magnetic Field", Int. J. Theor Phys, 55: (2016), http://dx.doi.org/10.1007/s10773-015-2905-7.

[2] D. A. M. Abo-Kahla, "The Pancharatnam Phase of a Three-Level Atom Coupled to Two Systems of N-Two Level Atoms", Journal of Quantum Information Science, 6: (2016), http://dx.doi.org/10.4236/jqis.2016.61006.

[3] G. Sadiek, E. Lashin, M. Sebawe Abdalla, " Entanglement of a two-qubit system with anisotropic XYZ exchange coupling in a nonuniform time-dependent external magnetic field", Physcia B, 404: (2009) 1719-1728, https://doi.org/10.1016/j.physb.2009.02.011

[4] Dae Mann Kim, Introductory Quantum Mechanics for Applied Nanotechnology, Weinheim, (2015), German.

[5] M. Abdel-Aty, M. Sebawe Abdalla, A.-S.F. Obada," Degree of entanglement for two fields interacting with a two-level system", Physica A, 307: (2002), https://doi.org/10.1016/S0378-4371(01)00629-X 
[6] M. Abdel-Aty, M. Sebawe Abdalla, A.-S.F. Obada," Entropy squeezing of a two-mode multiphoton Jaynes-Cummings model in the presence of a nonlinear medium", J. Opt. Quantum Semiclass. B, 4: (2002), https://doi.org/10.1088/1464-4266/4/2/309

[7] M. Abdel-Aty, M.Sebawe Abdalla, A.-S.F. Obada, "Entropy and phase properties of isotropic coupled oscillators interacting with a single atom: one-and two-photon processes", J. Opt. Quantum Semiclass. B, 4: (2002), https://doi.org/10.1088/14644266/4/3/367

[8] M. Sebawe Abdalla, M.M.A. Ahmed, A.-S.F. Obada, "The interaction between a single two-level atom coupled to the an-level quantum system through three couplings", Annals of Physics, 364: (2016), https://doi.org/10.1016/j.aop.2015.10.023

[9] M. Sebawe Abdalla, A.-S.F. Obada, E.M. Khalil, M.M.A. Ahmed, "Quantum treatment for three waves mutually interacting with a single two-level atom", Laser Phys., 24: (2014), https://doi.org/10.1088/1054-660X/24/10/105205

[10] Reda. M. El-Shishtawy, K. Berrada, Robert. C. Haddon, Yas F. Al-Hadeethi, Saleh. H. AlHeniti, Bahaaudin M. Raffah, "Quantum Transfer Energy and Nonlocal Correlation in a Dimer with Time-Dependent Coupling Effect", Int J Theor Phys., 56: (2017), https://doi.org /10.1007/s10773-017-3282-1

[11] A.-S. F. Obada, M. M. A. Ahmed, Ahmed M. Farouk, "A moving three-level -type atom in a dissipative cavity", Phys. J. D, 71: (2017), https://doi.org /10.1140/epjd/e2017-803575

[12] M. J. Faghihi, M. K. Tavassoly, M. R. Hooshmandasl, "Entanglement dynamics and position-momentum entropic uncertainty relation of a -type three-level atom interacting with a two-mode cavity field in the presence of nonlinearities", J. Opt. Soc. Am. B, 30: (2013), https://doi.org /10.1364/JOSAB.30.001109

[13] N.H. Abd El-Wahab, A. Salah, A. S. A. Rady, A.-N. A. Osman, "Entanglement Dynamics of a Three-level Atom in a Momentum Eigenstate Interacting with Non-linear Effect", Differ. Equ. Dyn. Syst. (2016).

[14] A.-S. F. Obada, M. M. A. Ahmed, Ahmed M. Farouk, "The Dynamics of a Five-level (Double)-type AtomInteracting with Two-mode Field in a Cross Kerr-like medium", Int. J. Theor. Phys, 57: (2018), https://doi.org/10.1007/s10773-017-3651-9

[15] D. A. M. Abo-Kahla, M.M.A. Ahmed, A. T. M. Makram-Allah, "Analytical solution of a three-level atom coupled to four systems of N-two-level atoms", Optical Review, 26: (2019), https://doi.org/10.1007/s10043-019-00559-7.

[16] K. D.-Peng, L. Q.-Hong, A. M. Ashfaqb, W. Y.-Yuan, L. S.-Tian, "Entropy squeezing of an atom with a k-photon in theJaynesCummings model", Chin. Phys. B, 19: (2010) 014206.

[17] H. R. Baghshahia, M. K. Tavassolya, A. Behjata, "Entropy squeezing and atomic inversion in the $\kappa$-photon Jaynes-Cummings model in the presence of the Stark shift and a Kerr medium: A full nonlinear approach", Chin. Phys. B, 23: (2014) 074203.

[18] E. Faraji, H. R. Baghshahi, M. K. Tavassoly, "The influence of atomic dipole-dipole interaction on the dynamics of the population inversion and entanglement of two atoms 
interacting non-resonantly with two coupled modes field", Mo. Physics Letters B, 31: (2017) 1750038.

[19] M. J. Faghihi, M. K. Tavassoly, and M. Hatami, "Dynamics of entanglement of a threelevel atom in motion interacting with two coupled modes including parametric down conversion", Physica A, 407: (2014) 100.

[20] M. Youssef, A.-S. F. Obada and N. Metwally, "Some entanglement features of three atoms Tavis- Cummings model: Cooperative case", J. Phys. B: At. Mol. Opt. Phys., 43: (2010) 095501.

[21] E. K. Bashkirov, "Dynamics of the Two-Atom Jaynes-Cummings Model with Nondegenerate Two-Photon Transitions", Laser Phys.,16: (2006) 1218.

[22] M. O. Scully, M. S. Zubairy, Quantum Optics, (1997), Cambridge University.

[23] H. Y.-XIA, G. G.-CAN, "A new measure for the Jaynes-Cummings model dynamics", Chin. Phys., 5: (1996) 901.

[24] Q. Liaoa, G. Fanga, Y. Wangc, M. A. Ahmad, S. Liu, "Single-atom entropy squeezing for two two-level atoms interacting with a binomial field", Optik., 122: (2011) 1392.

[25] F. A. A. El-Orany, M. R. B. Wahiddin, A.-S. F. Obada, "Single-atom entropy squeezing for two two-level atoms interacting with a single-mode radiation field", Opt. Commun., 281: (2008) 2854-2863.

[26] M. Sebawe Abdalla, M.M.A. Ahmed, "Some statistical properties for a spin (1/2) particle coupled to two spirals", Opt. Commun., 285: (2012), https://doi.org/10.1016/j.optcom.2012.04.014.

[27] A.-S.F. Obada, M. Abdel-Aty, "Nonclassical effects in a three-level atom one-mode system with arbitrary forms of nonlinearities", Physica A, 329: (2003) 53.

[28] Gerry Christopher, Knight Peter, Introduction to Quantum Optics, (2004), Cambridge University.

[29] Ficek Zbigniew, Wahiddin Mohamed Ridza, Quantum Optics for Beginners, (2014).

[30] R. Glauber, "Photon correlations", Phys. Rev. Lett., 10: https://doi.org/10.1103/PhysRevLett.10.84

(1963),

[31] R. Hanbury Brown, R. Q., "Twiss Correlation between photons in two coherent beams of light", Nature, 177: (1956), https://doi.org/10.1038/177027a0

[32] D. F. Walls, G. J. Milburn, Quantum Optics, (1994), Springer.

[33] E. Dibakar, A. Karimi, M. K. Tavassoly, "Excitation and depletion of entangled squeezed states their properties and generation", Phys. Scr.,90: (2015) 085102.

[34] N. H. Abdel-Wahab, M. F. Mourad, "On the interaction between two two-level atoms and a two-mode cavity field in the presence of detuning and cross-Kerr nonlinearity", Phys.Scr., 84: (2011) 015401. 
[35] A.-S. F. Obada, M. M. A. Ahmed, Ahmed M. Farouk, "The Dynamics of a Five-level (Double $\Lambda$ )-type Atom Interacting with Two-mode Field in a Cross Kerr-like Medium", Int. J. Theor. Phys, 57: (2018), https://doi.org/10.1007/s10773-017-3651-9.

[36] A.-S. F. Obada, M. M. A. Ahmed, S. Sanad, "Non-classical properties for SU $(1,1)$ pair coherent states", J. Phys. Commun., 4: (2020), https://doi.org/10.1088/2399-6528/ab66d1

[37] A.-S. F. Obada, M. M. A. Ahmed, S. Sanad, "Quantum Properties for a State of N Twolevel Atoms", International Journal of Theoretical Physics, 58: (2019), https://doi.org/10.1007/s10773-019-04147-y.

[38] M. Y. Abd-Rabbou, E. M. Khalil, M. M. A. Ahmed, A.-S. F. Obada, "External Classical Field and Damping Effects on a Moving two Level atom in a Cavity Field Interaction with Kerr-like Medium", International Journal of Theoretical Physics, 58: (2019), https://doi.org/10.1007/s10773-019-04268-4.

[39] A.-S. F. Obada, M. M. A. Ahmed, M. Abu-Shady, H. F. Habeba, "Effect of an External Magnetic Field on Some Statistical Properties of the 2+1 Dirac-Moshinsky Oscillator", J. Russ Laser Res, 40: (2019), https://doi.org/10.1007/s10946-019-09808-1.

[40] A.-S. F. Obada, M. M. A. Ahmed, M. Abu-Shady, H. F. Habeba, "Interaction Between $\mathrm{Su}(1,1)$ Quantum System and a Three-Level Atom in the Presence of the Stark Shift". https://www.researchgate.net/publication/339291025.

[41] D. A. M. Abo-Kahla, "Information entropy and population inversion of a three-level semiconductor quantum dot", Indian J. Phys., https://doi.org/10.1007/s12648-020-018143.

[42] Q.-C. Zhou, S.N. Zhu, "Dynamics of a single-mode field interacting with a K-type threelevel atom", Opt. Commun. 248: (2005) 437

[43] M. Abdel-Aty, "Quantum Phase Entropy and Entanglement of a Multiphoton ThreeLevel Atom near the Edge of a Photonic Band Gap", Laser Phys. 16: (2006) 1381.

[44] D. A. M. Abo-Kahla, "The atomic inversion and the purity of a quantum dot two-level systems", Applied Mathematics \& Information Sciences, 10: (2016) 1-5.

[45] D. A. M. Abo-Kahla, M. Abdel-Aty, A. Farouk, "The Population Inversion and the Entropy of a Moving Two-Level Atom in Interaction with a Quantized Field", International Journal of Theoretical Physics, (2018) 1-11.

[46] Clive Emary, Christina P"oltl, Alexander Carmele, Julia Kabuss, Andreas Knorr, Tobias, "Brandes Bunching and anti-bunching in electronic transport", Institut fur Theoretische Physik, Hardenbergstr. 36: (2012) TU Berlin, D-10623 Berlin, Germany. 
التفاعل بين ذرة ثلاثية المستويات وأربعة أنظمة تحتوي على ن من الذرات ثنائيه المستوى

أسماء طارق محمد عبد اللطيف مكرم الله

معيدة بقسم الرياضيات (الرياضيات التطبيقية)

كلية البنات - جامعة عين شمس لرس

أ.د. محمد محمد علي أحمد

أستاذ الرياضيات التطبيقيه

كلية العلوم - جامعة الأزهر

أ.د.م. دعاء أحمد محمد أبو كلة

أستاذ الرياضيات التطبيقية المساعد

كلية التربية - جامعة عين شمس لرسباد

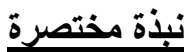

لدينا أربعة أنظمة، كل منها يحتوي على ن من الذرات، وكل ذرة منها تتكون من مستويين، وكلها تتفاعل مع ذرة تتكون من ثلاثة مستويات. علاوة على ذلك، ترتبط الذرة أيضًا بالأنظمة من خلال معاملات الاقتران التي تعتمد على الزمن. من خلال حالات خاصة، يتم الحصول على الحل الدقيق للدالة الموجة. ثم نقوم بدر اسة الانقلاب الذري بالإضافة إلى الارتباط الطبيعي.

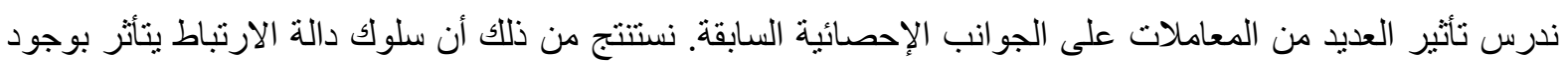
معاملات الاقتران التي تعتمد على الزمن بين الدورات. لذللك، يمكننا التحكم في هذا النموذج من خلال هذه المعاملات. 